\title{
Note sur le canicide chez les Kabiyé du Nord-Togo
}

A note on killing dogs among the Kabre in North Togo

\section{Raymond Verdier}

\section{OpenEdition}

\section{Journals}

Édition électronique

URL : http://journals.openedition.org/span/215

DOI : 10.4000/span.215

ISSN : 2268-1558

\section{Éditeur}

École pratique des hautes études. Sciences humaines

\section{Édition imprimée}

Date de publication : 1 septembre 1975

Pagination : 129-136

ISSN : 0294-7080

\section{Référence électronique}

Raymond Verdier, « Note sur le canicide chez les Kabiyé du Nord-Togo », Systèmes de pensée en Afrique noire [En ligne], 1 | 1975, mis en ligne le 08 juillet 2013, consulté le 19 avril 2019. URL : http:// journals.openedition.org/span/215; DOI : 10.4000/span.215 
NOTE SUR LE CANICIDE CHEZ LES KABIYE DU NORD-TOGO

par Raymond VERDIER

RESUME - Le chien participe à la fois du monde sauvage et du monde domestique. Cette situation ambivalente est marquée, tant dans la littérature orale (mythes, contes) que dans le tituel initiatique de la classe d'âge des efala, par le lien étroit entre le chien et la nort; ce lien est souligné à deux niveaux, d'abord au plan de la création divine où le chien est le signe de la condition mortelle de l'homme, ensuite au plan de l'initiation ou sa mort par etouffement est le signe du passage de l'enfance à l'adolescence.

Nombreux sont les paysans kabiyé qui élèvent actuellement des chiens et en font le commerce tant sur les marchés extérieurs qu'intérieurs.

Jadis, quand la montagne etait couverte de forêts, la chasse jouait un rôle très important sur le plan économique et il y a lieu de penser qu'au temps de son implantation dans la région, il y a plusieurs siècles, le Kabiyé tetait principalement un chasseur et secondairement un cultivateur. Le chien $y$ était alors l'indispensable compagnon de l'homme qu'il suivait à la chasse.

Avec le déboisement progressif des collines, l'activité agricole a pris peu à peu le pas sur 1'activité cynégêtique et le Kabiyé est à l'heure actuelie avant tout un agriculteur; la chasse est devenue une activité marginale, la viande du gibier, une nourriture d'appoint et le chien, le gardien du foyer domestique.

Ces transformations de l'économie devaient être sommairement évoquées pour comprendre l'importance que tient le chien tant dans la mythologie que dans le rituel initiatique. 
I Le chien dans les mythes et les contes.

Dans le récit de la création, l'homme est chassé du lieu palsible où Dieu l'a fait descendre; sous la poursuite des hiboux, il doit quitter sa "terre humide" et va désormais travailler et mourir. Chassé, il devient chasseur; avec la construction de l'arc et la fabrication du poison, c'en est fini de la paix fraternelle entre les hommes, la mort règne desormais; l'âge d'or a pris fin avec l'avènement de la chasse et de la guerre.

L'activité cynégétique est dès lors le lot de l'homme, condamné à tuer pour vivre et destiné à mourir : le chien sera à la fois son compagnon nécessaire et le signe de sa condition mortelle.

Ce lien entre le chien et la condition mortelle de l'homme est très fortement marqué dans les deux contes suivants :

LE CHIEN ET LE SINGE

Narrateur : TATA Norbert village Yade.

1. Jadis, Dieu chargea quelqu'un d'aller sur la terre annoncer aux homes que personne ne mourra.

2. L'envoyé en chemin rencontra l'arbre à néré et y resta pour manger la farine.

3. Dieu, ne le voyant pas revenir, demanda au chien d'aller annoncer la nouvelle aux hommes.

4. Le chien courut au pays des hommes et leur annonça qu'ils devaient mourir.

5. Là-dessus, le premier envoyé arriva et déclara que désormais personne ne meurt. 
6. Les hommes rêpliquèrent que le chien leur avait annonce qu'ils mourraient.

7. Le chien retourna auprès de Dieu; Dieu l'appela singe et le renvoya au pays des hommes pour aller vivre dans la brousse.

8. C'est ainsi que maintenant les hommes meurent et le singe est devenu une bête de la brousse.

LE CHIEN ET LA MORT DE L'HOMME

Narrateur : E. BASSARI village Farendé

1. Un jour, Dieu appela tous les animaux domestiques et les interrogea sur la destinée des êtres humains.

2. Les animaux se mirent en route; le chien courut et les dépassa.

3. En chemin, il trouva un os, mais le laissa et continua sa route ; la chèvre, qui avait pris de l'avance au départ, s'attarda à manger le fruit du néré.

4. Le chien arriva le premier auprès de Dieu : désires-tu, lui demanda-t-il, que l'homme meure ou non ? Qu'il meure répondit le chien.

5. Alors Dieu lui posa cette question : qui te nourrit ? - le chien : "ça sort du foyer !".

6. Il I'interrogea encore : Veux-tu que l'homme ressuscite? - le chien : "qu'il meure à jamais".

7. Quand la chèvre arriva, Dieu le questionna pareillement, - la chèvre : "qu'il meure et ressuscite; ma vie dépend d'une fillette qui me garde et s'occupe de moi".

8. Le mouton fit la même réponse.

9. Ia poule : "je dépends de celui qui monte au grenier". 
10. Le premier homme mort ressuscita selon la parole de la chèvre, du mouton et de la poule mais il n'avait pas de nez et était squelettique.

11. C'est à cause du chien que les hommes meurent et ne ressuscitent pas.

La chienne condition revient désormais à l'homme; comme le chien réduit à la vie de brousse, l'homme se trouve placé à l'exterieur de l'univers divin; il n'est plus au dedans, il est au dehors de la création.

Mais le chien, symbole de la dégradation de l'homme séparé de son crêateur et menant une existence sauvage dans la brousse, apparaît d'un autre côté comme l'animal domestique par excellence, qui figure l'état de l'homme civilisé.

Grâce au chien, l'homme va accéder à son humanité ; c'est en effet le chien qui va permettre a l'homme de ne plus manger crusses aliments en dérobant le feu :

\section{LE CHIEN ET LE FEU}

$$
\begin{array}{r}
\text { Narrateur: Norbert TATA } \\
\text { village Yadé. }
\end{array}
$$

1. Jadis il n'y avait pas de feu dans le village, les hommes mangeaient la nourriture crue.

2. Un jour, ils ont entendu dire que le feu se trouvait sur une grande montagne, sous la garde des lions.

3. Les hommes ne savaient pas qui envoyer lä-bas ; finalement ils choisirent le chien.

4. Le chien partit, arriva en I'absence des lions et prit le feu ; sur le chemin du retour, il urinait à chaque pas.

5. Les lions, quand ils furent rentrés, constatêrent que quelqu'un était venu chercher le feu et ils dirent au feu d'aller brûler le voleur. 
6. Le feu se leva, brûla toút jusqu'au bord du chemin, où le chien avait uriné.

7. Le chien arriva à la maison et déposa le feu dans le vec-tibule.

8. A partir de ce jour, tout le monde allait y chercher le feu et le feu se répandit dans tout le village.

9. Alors on prit le chien pour le déposer près du feu; le feu lui appartient et c'est la raison pour laquelle le chien reste auprès du foyer.

Maftre du feu, l'homme va désormais respecter la vie de ses semblables; il ne lui sera plus nécessaire de sacrifier la vie des siens pour triompher de la mort : le fidèle compagnon prend la place de l'homme, il devient son substitut dans le sacrifice, comme il est dit dans le conte suivant :

\section{LE CHIEN ET LA FEMME STERIIE}

\section{Narrateur : KANDO village Pagouda.}

1. Une femme était stérile ẹt c'est pourquoi on 1 'amena sous "1'arbre". pour être fécondée.

2. L'homme, qui l'amena au lieu lui dit : si tu as un enfant, tu l'apporteras sous l'arbre.

3. A son retour, elle fut enceinte; elle mit au monde une fille.

4. Quand la fille fut grande, elle chercha un amant; elle lui dit : mon père avait amené ma mère sous "l'arbre" et je suis destinée à être sacrifiée en récompense de la fécondité donnée à ma mère.

5. Le jeune homme lui dit : est-il possible qu'on sacrifie ainsi une vie humaine ! Si tu dois aller sous l'arbre, je t'accompagnerai avec de la farine et un chien.

6. Quand les parents s'en vinrent sous l'arbre avec leur fille, ils la placèrent à l'endroit oũ elle devait être sacrifiée. 
7. Le jeune homme, qui etait cache dans l'arbre, descendit et leur dit : "Cela ne se fait pas; on vient ici pour trouver la santé, va-t-on sacrifier une personne! Sacrifiez ces pouleset, si cela ne réussit pas, tuez ce chien ".

8. On sacrifia les poules, puis le chien.

9. Jadis on sacrifiait des personnes; aujourd'hui on ne tue plus les hommes sous l'arbre, parce que l'on tue des chiens à leur place.

10. Si un homme âgé décède, on tue un chien à ses funerrailles avant de tuer des boeufs.

11. L'homme mange le chien, mais les femmes n'en mangent pas car elles seraient souillées.

Ce dernier conte introduit un nouvel attribut du chien se rapportant à la souillure ; avant d'y revenir à propos du rituel, nous pouvons rapporter les diverses fonctions et rôles du chien dans la mythologie et les contes à son association fondamentale à la mort et à sa position ambivalente en tant qu'animal relevant à la fois de l'orare sauvage et de l'ordre domestique :

\section{Monde sauvage}

messager de la mort

cru

bête de brousse

interdit de l'impurete

\section{Monde domestigue}

voleur du feu culturel cuit

gardien $\mathrm{du}$ foyer sacrifice, substitut del'hame

\section{II - Le chien dans le rituel initiatique.}

C'est par l'intermédiaire de la chasse et du chien que s'est accompli le passage de l'enfance de l'humanité (âge d'or) à I'âge de notre humanité présente ; de même, c'est par l'intermédiaire de la chasse et du chien que s'effectue le passage de l'enfance à l'adolescence dans la vie humaine. 
La mort à l'enfance marque l'avènement de la condition de chasseur; le rituel d'initiation des adolescents de 15 à 18 ans est centré sur la chasse et le chien : le garçon nouveauné efalo va devoir apprendre à chasser et manger pendant trois ans du chien.

A chaque garçon initie, il faut un chien; la mort d'un chien est la condition et le signe d'une nouvelle naissance. Il incombe à l'oncle maternel de se procurer un chien pour que l'initiation de son neveu puisse avoir lieu ; s'il n'en a pas les moyens et $\mathrm{si}$ le père ne peut l'aider, l'initiation du garçon sera reportée à l'année suivante.

Capturé en janvier, l'enfant est tatoué et fait une retraite de case de six jours; un poulet est sacrifié sur l'autel de son wayitu, "la pâte des larmes" est consommée et 1'on immole un bélier sur l'autel familial.

Quand la retraite prend fin, le garçon se rend en brousse en compagnie des autres initiés et c'est alors la tuerie des chiens : chaque efalo doit étrangler le chien qui lui a été destiné; l'animal ne peut être égorgé, il doit être étouffé par strangulation. Après l'hécatombe, les initiés tenant leur animal par le cou à bout de bras, se rendent en dansant au marché et à partir de ce moment, leur nouvelle condition est connue de tous. Puis ils se rendent à l'écart du village au milieu des rochers pour manger le chien; on brûle ses poils, on le dépèce et on enterre ses intestins, on fait cuire sa chair dans une marmite spécialement consacrée à cet usage et on grille enfin les morceaux au feu.

Ce repas est la marque même de l'initiation qui se dit : efalo tohsu, "faire manger l'efalo"; l'initié est alors considéré corme impur ; $i l$ ne pourra rentrer au village qu'après s'être lavé au marigot. Durant les trois ans de l'initiation où il va manger du chien, il doit s'abstenir de tout rapport sexuel.

Hayisu, littéralement "faire trouver le chien", dësigne la course de fond qui précède la première grande chasse en février. L'efalo gagnant sera l'arbitre de la chasse en cas de contestation. 
A la saison des pluies, en juillet, les e fala vont devoir lutter entre eux, d'abord par quartier, puis par village, enfin entre villages du même canton; le corps jadis enduit de la graisse de chien, les lutteurs, torse nu, s'affrontent un $\precsim$ un : il faut renverser l'adversaire et le plaquer, le dos contre terre; le vainqueur $n$ 'est pas nécessairement le plus fort mais le plus habile et rusé.

Les efala lutteront encore les deux années suivantes avec leurs camarades de promotion jusqu'à leur entrée dans la classe supérieure, celle des sangayin, les purifiés. Ils cesseront désormais de manger le chien : l'interdit sexuel est alors levé.

0

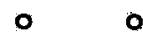

Ainsi le chien se trouve lié à la mort au double plan de la création et de l'initiation :

Création

Vie------>Mort
Initiation

Mort------ $\rightarrow$ Vie

Dans les deux cas, la chasse marque la transition d'un état à l'autre; le chien, animal possédant la double vue, kenao, et participant à la fois du monde sauvage et du monde domestique, symbolise ce passage conflictuel.

C'est sans doute cette position particulière qui le rend impur et a pour effet, d'un côté d'interdire sa consommation aux femmes, de l'autre d'être consommé lors de l'initiation sans effusion de sang, hors du village et hors de tout rapport sexuel. 